\title{
Internal exposure in post-accidental situations: a multi-pollution to take into account?
}

\author{
Jean-Marc Bertho ${ }^{1, *}$, Stefania Musilli ${ }^{1}$, and Karine Tack $^{1}$ \\ ${ }^{1}$ IRSN, PSE-SAN/SESANE, LRTOX, BP 17, 92262 Fontenay aux roses cedex, France
}

\section{Introduction}

Major nuclear accidents (Chernobyl, Fukushima) induced the contamination of large areas with a mix of radionuclides (RNs). On the long term the exposure of the population is limited to some key RNs such as ${ }^{137} \mathrm{Cs},{ }^{134} \mathrm{Cs}$ and ${ }^{90} \mathrm{Sr}$ and to external exposure at low dose and low dose rate.

Numerous studies, either epidemiological or more limited, were conducted on the health effects of long term chronic ingestion of RNs. Epidemiological studies showed the occurrence of leukemia both in liquidators and in the general population. Other somatic pathologies were also evidenced such as cardiovascular diseases and cataracts. More limited studies showed the occurrence of other somatic disorders such as changes in the immune system in human population living on these contaminated territories. In numerous cases, the observed effects were ascribed to the internal contamination with ${ }^{137} \mathrm{Cs}$. However, the direct link with ${ }^{137} \mathrm{Cs}$ internal exposure or with external exposure was not always clearly established. Moreover, in most of epidemiological studies, the assessment of exposure in post-accidental situation remains difficult with numerous uncertainties. As a result, the health effects of incorporated radionuclides by populations living in a contaminated territory remain a matter of controversy.

\section{Experimental studies in animal models.}

In order to overcome the uncertainties in exposure assessment, several groups studied the effect of chronic ingestion of radionuclides in rodents, with various schedule of exposure, either acute or chronic. Since ${ }^{137} \mathrm{Cs}$ is the most frequent $\mathrm{RN}$ found in the environment, this $\mathrm{RN}$ was the most studied. After an acute exposure, it was shown the appearance of an hematopoietic syndrome in a dose dependant manner. As well, the frequency of tumors was increased according to the ${ }^{137} \mathrm{Cs}$ dosage. Studies of chronic exposures through ingestion showed the appearance of numerous biological effects, both in the rat and in the mouse models. However most of these effects were of limited amplitude and did not led to the appearance of pathologies. This was the case for instance for the oxysterol metabolism with changes evidenced in steroid hormone synthesis, Vitamin D metabolism and hepatic cholesterol metabolism, and for the sleep wake cycle. The main change observed was a change in the cardiovascular system with a decrease in the arterial pressure and a disappearance of the circadian rhythm of this AP.

* Corresponding author: jean-marc.bertho@irsn.fr 
These experimental results are somewhat in contradiction with results from the study of human populations. In fact, there is no evidence of a cardiovascular dysfunction induced by exposure to ${ }^{137} \mathrm{Cs}$ in the general population. As well, there are numerous studies in humans exposed to ${ }^{137} \mathrm{Cs}$ showing alterations in the immune system while in the rodent model, no change was evidenced.

A possible hypothesis to explain these discrepancies is the uncertainties in the exposure of populations in a post-accidental exposure. For instance, ${ }^{90} \mathrm{Sr}$, which is also present in the environment of territories contaminated by the Chernobyl accident, was recently shown to induce changes both in bone physiology and in the immune response induced by an antigen in a vaccine model in rodents. These results are in accordance with results of large studies in the population living on the riverside of the Techa River, heavily contaminated with ${ }^{90} \mathrm{Sr}$, among other radionuclides. Of course, these results demonstrating health effects of ${ }^{90} \mathrm{Sr}$ chronic ingestion cannot explain all of the health effects of chronic long term exposure in a post accidental situation. However, these data strongly suggest that this multiple exposure, including external irradiation at low dose rate and internal exposure with several RNs should be taken into account in future studies.

\section{Radiological and chemical multiple exposures.}

There is almost no available study about the health effects of a combination of RNs with either a chronic or an acute exposure. In fact, the ICRP consider the additivity of radiation doses, whatever their origin. This principle is in accordance with the direct proportionality between the frequency of stochastic effect and the radiation exposure. However, this principle does not take into account several points. The first one is the chemical toxicity of stable nuclides, which may become preponderant at low concentrations, as it is the case for Uranium. The second point is the description of non-linear effects at low doses such as bystander effects, hormesis or adaptative effects. And the third point is the existence of other chemical pollutants in the environment that may interfere with ionizing radiation effects, especially at low doses. For instance, it is well known that radon exposure exacerbate the risk of lung cancers in smokers as compared to non-smokers. As well a recent study showed an increased risk of ${ }^{131}$ I-induced thyroid cancer if there is a coexposure to excess of nitrates.

\section{Conclusions.}

The hypothesis of a more generalized model of interactions between chemical pollutants and radiological pollutants is reinforced by the recent descriptions of non-additive (either supra- or infra-) biological effects of mixtures of chemical pollutants. For instance, a study of 7 different combinations of 2-6 chemical pollutants showed in some cases supra additive effects that were not predicted on the basis of toxicological reference values. Since there is no available study on the health effects of mixtures of radionuclides, it is not possible to exclude the hypothesis that a mixture of RNs may induce non-linear, and possibly supra additive effects as compared to the effect of individual RNs. Thus it appears that futures studies on the health effects of radionuclides at low concentrations should be done taking into account more realistic schemes of exposure, including several radionuclides, but also mixtures of RNs and chemical pollutants. Such mixtures may explain some of the currently observed health effects of exposure in post accidental situations. 